\title{
Modelagem computacional aplicada à flambagem elástica de colunas
}

Computational modeling applied to the elastic buckling of columns

\author{
R. C. de A. Alonso ${ }^{1 *}$, D. S. de Lima ${ }^{2}$, M. Gomes ${ }^{2}$, M. de V. Real ${ }^{1,2}$, E. D. dos \\ Santos $^{1,2}$, L. A. Isoldi ${ }^{1,2}$ \\ ${ }^{I}$ Programa de Pós-Graduação em Modelagem Computacional (PPGMC), Universidade Federal do Rio Grande \\ (FURG), 96203-900, Rio Grande-RS, Brasil \\ ${ }^{2}$ Escola de Engenharia (EE), Universidade Federal do Rio Grande (FURG), 96203-900, Rio Grande-RS, Brasil \\ *renatalonso@yahoo.com.br
}

(Recebido em 15 de setembro de 2014; aceito em 29 de dezembro de 2014)

Flambagem é um fenômeno de instabilidade que ocorre em elementos estruturais esbeltos submetidos à carregamento de compressão. A flambagem elástica é caracterizada por uma repentina deflexão lateral do membro estrutural esbelto, quando a carga crítica de flambagem é atingida. Em colunas a flambagem elástica é considerada o colapso da estrutura. O objetivo desse trabalho é apresentar três diferentes modelos computacionais para tratar a flambagem elástica de colunas. O software ANSYS, que é baseado no Método dos Elementos Finitos (MEF), foi adotado para desenvolver esses modelos, empregando os elementos BEAM189, SHELL93 e SOLID45. As condições de contorno e a carga compressiva devem ser aplicadas de maneira específica em cada modelo, dependendo das características do elemento finito. Diferentes condições de contorno foram consideradas para cada modelo numérico. Para realizar a verificação desses modelos, a solução analítica para essas condições de contorno foram usadas. Todos os resultados numéricos obtidos estão em concordância com os analíticos, sendo a maior diferença encontrada de $2,83 \%$. Além disso, as formas flambadas das colunas obtidas numericamente também estão de acordo com as configurações teóricas. A vantagem para o uso dos modelos computacionais desenvolvidos com os elementos finitos SHELL93 e SOLID45 é a possibilidade de analisar colunas com furos ou com geometrias complexas.

Palavras-Chaves: Flambagem, Carga Crítica, Simulação Numérica

Buckling is an instability phenomenon which occurs in slender structural members subjected to compressive load. The elastic buckling is characterized by a suddenly lateral deflection of the slender structural member, when the critical load is reached. In columns the elastic buckling is considered the collapse of the structure. The goal of this work is to present three different computational models to analyze the elastic buckling of columns. The software ANSYS, which is based on the Finite Element Method (FEM), was adopted to develop these models, employing the elements BEAM189, SHELL93 and SOLID45. The boundary conditions and compressive load must be applied in a specific way for each model, depending of the finite element characteristics. Different boundary conditions were considered for each numerical model. In the verification of these models, the analytical solutions for these boundary conditions were used. All the numerical results obtained are in agreement with the analytical ones, being the higher difference encountered of $2.83 \%$. Besides, the columns buckled shapes numerically obtained are also in agreement with the theoretical configurations. The advantage of the use of computational models developed with SHELL93 and SOLID45 finite elements is the possibility of analyzing columns with holes or with complex geometries.

Keywords: Buckling, Critical Load, Numerical Simulation

\section{INTRODUÇÃO}

Sempre que se projeta um elemento estrutural, é necessário que ele satisfaça requisitos específicos de resistência, deflexão e estabilidade [1]. O foco desse trabalho está relacionado a um fenômeno de instabilidade estrutural chamado flambagem. Uma estrutura elástica estável será submetida deslocamentos proporcionais ao carregamento aplicado: um pequeno aumento no carregamento ocasionará um pequeno aumento no deslocamento. Portanto a instabilidade estrutural acontece quando um pequeno incremento no carregamento causa um grande deslocamento na estrutura [2]. 
Em elementos estruturais sujeitos à compressão, sendo estes esbeltos e compridos, a carga poderá ser grande o suficiente para provocar uma deflexão lateral: a flambagem. A flambagem de uma coluna pode resultar na falha repentina de uma estrutura ou mecanismo e por isso devese evitar que esse fenômeno de instabilidade estrutural aconteça [1]. Para isso é necessário conhecer a carga crítica de flambagem da coluna, que pode ser entendida como a carga axial máxima que o componente estrutural pode suportar quando está na iminência de flambar, ou seja, qualquer carga adicional provocará deflexão lateral.

A carga crítica de uma coluna pode ser determinada experimentalmente, analiticamente ou numericamente. O objetivo do presente trabalho é apresentar três diferentes modelos computacionais para a determinação da carga crítica de colunas. Para isso foi empregado o software ANSYS, que é baseado no Método dos Elementos Finitos (MEF), sendo a verificação dos modelos desenvolvidos feita através da comparação entre o resultado numérico e a solução analítica. A diferença entre os três modelos computacionais propostos consiste no tipo de elemento finito empregado na discretização do problema. Elementos finitos tipo viga (BEAM189), casca (SHELL93) e sólido (SOLID45) foram usados, dando origem aos três modelos computacionais desenvolvidos.

\section{FLAMBAGEM DE COLUNAS}

As colunas esbeltas quando submetidas à compressão estão sujeitas à ocorrência de um tipo de comportamento mecânico conhecido como flambagem. Enquanto a carga sobre esse membro é relativamente pequena, a coluna fica submetida a uma tensão de compressão e consequentemente a uma deformação axial. Porém, existe um valor crítico para esse carregamento de compressão no qual a coluna repentinamente sofre uma deflexão lateral, ocorrendo a flambagem e dando origem a grandes deformações que causam o colapso da coluna. Portanto, essa carga crítica a partir da qual a flambagem ocorre é um critério de projeto que deve ser considerado para colunas, uma vez que a intensidade dessa carga normalmente é muito menor que a necessária para atingir a tensão de escoamento do material [3]. Em outras palavras, de acordo com Hibbeler [1], a carga crítica é a carga axial máxima que uma coluna pode suportar antes de ocorrer a flambagem. Qualquer carga adicional provocará flambagem na coluna, conforme mostra a Figura 1.

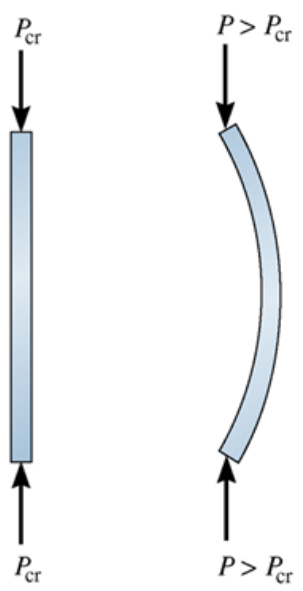

Figura 1: Coluna sob Flambagem [1].

Para compreender melhor esse tipo de instabilidade, é interessante considerar um mecanismo formado por duas barras sem peso, rígidas e acopladas por pinos nas duas extremidades conforme Figura 2. 


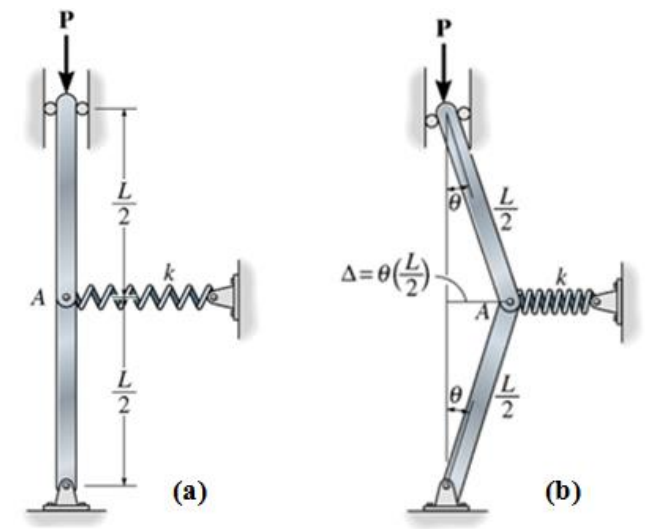

Figura 2: Modelo formado por um mecanismo de barras [1].

Observando a Figura 2, nota-se que inicialmente as barras estão perfeitamente alinhadas e submetidas a uma pequena força compressiva (Figura 2a), estando o sistema em equilíbrio enquanto não ocorrerem perturbações. Porém, se o ponto $A$ for ligeiramente deslocado para direita, de maneira que cada barra forme com a vertical um pequeno ângulo $\theta$ em relação à posição inicial (Figura 2b), é possível afirmar que ocorreu uma perturbação no sistema. Então, se após este deslocamento do ponto $A$ o sistema retornar à posição de equilíbrio este é chamado de estável:

$$
P<\frac{k L}{4}
$$

condição na qual a força desenvolvida pela mola seria adequada para devolver as barras às suas respectivas posições verticais. Caso o sistema continue se movendo para fora da posição de equilíbrio, o sistema é chamado de instável:

$$
P>\frac{k L}{4}
$$

e, portanto, o mecanismo tenderá a sair do equilíbrio e não retornar à sua posição original. A última possibilidade é que ocorra um equilíbrio neutro:

$$
P=\frac{k L}{4}=P_{c r}
$$

e nesse caso o valor da carga $P$ representa o valor da carga crítica $\left(P_{c r}\right)$. Fisicamente o valor de $P_{c r}$ define a carga sob a qual o mecanismo está na iminência de sofrer flambagem.

Com base no exposto é possível então definir a carga crítica para uma coluna ideal com apoios de pinos ou birrotulada (Figura 3).

A carga $P$ (Figura 3a) poderia ser aumentada até que o limite de escoamento ou de ruptura do material do qual a coluna é fabricada fosse alcançado. Entretanto, quando a carga crítica $P_{c r}$ é atingida, a coluna está no limite de tornar-se instável. Qualquer pequena redução de $P$ para um valor menor que $P_{c r}$ permite que a coluna fique reta e qualquer aumento de $P$ para um valor maior que $P_{c r}$, provoca aumento adicional da deflexão lateral. $\mathrm{O}$ fato da coluna permanecer estável ou tornar-se instável, quando submetida à carga axial de compressão, depende de sua habilidade de restauração que, por sua vez, é baseada em sua resistência à flexão. 


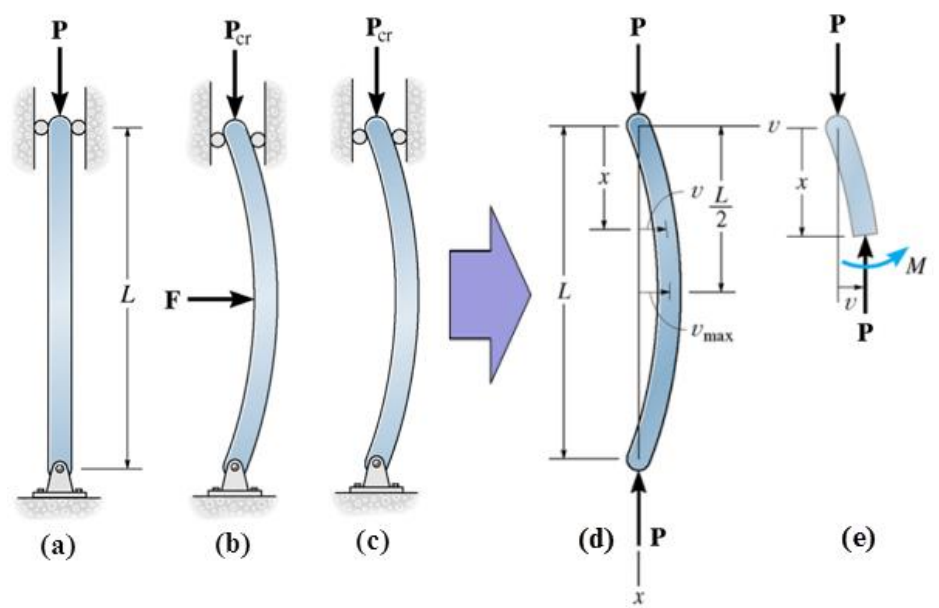

Figura 3: Flambagem de uma coluna com apoios de pinos [1].

Então, para determinar a carga crítica e a forma de flambagem da coluna, é necessário aplicar a equação diferencial da linha elástica que relaciona o momento fletor interno da coluna e sua forma fletida (Figuras $3 \mathrm{~d}$ e $3 \mathrm{e}$ ):

$$
E I \frac{d^{2} v}{d x^{2}}=M(x)=-P v \quad \text { ou } \quad \frac{d^{2} v}{d x^{2}}+\frac{P v}{E I}=0
$$

onde: $E$ é o módulo de elasticidade do material da coluna $\left(\mathrm{N} / \mathrm{m}^{2}\right) ; I$ é o momento de inércia da seção transversal da coluna $\left(\mathrm{m}^{4}\right) ; M$ é o momento fletor interno na coluna $(\mathrm{N} \cdot \mathrm{m})$, obtido através do método das seções (Figura 3e); $P$ é carga aplicada na coluna $(\mathrm{N})$; e $v$ é deflexão lateral da coluna (m). A Equação (4) é uma equação diferencial homogênea de segunda ordem com coeficientes constantes. Sua solução geral é dada por:

$$
v=C_{1} \operatorname{sen}\left(\sqrt{\frac{P}{E I}} x\right)+C_{2} \cos \left(\sqrt{\frac{P}{E I}} x\right)
$$

sendo $C_{1}$ e $C_{2}$ constantes de integração determinadas pelas condições de contorno nas extremidades da coluna (Figura 3a), que são: $v=0$ em $x=0$, obtendo $C_{2}=0$; e $v=0$ em $x=L$ definindo:

$$
C_{1} \operatorname{sen}\left(\sqrt{\frac{P}{E I}} L\right)=0
$$

A solução trivial para a Equação (6) seria $C_{1}=0$, porém nesse caso a deflexão seria nula $(v=0)$ para qualquer carga $P$ aplicada. Por isso é necessário impor uma solução não trivial:

$$
\operatorname{sen}\left(\sqrt{\frac{P}{E I}} L\right)=0
$$

A Equação (7) é satisfeita se:

$$
\sqrt{\frac{P}{E I}} L=n \pi \quad \text { ou } \quad P=\frac{n^{2} \pi^{2} E I}{L^{2}} \quad(n=1,2,3, \ldots)
$$

Na Equação (8), $n$ representa o número de semi-ondas na forma fletida da coluna e o menor valor de $P$ é obtido para $n=1$, definindo a carga crítica para uma coluna birrotulada, também chamada de carga de Euler, que é dada por:

$$
P_{c r}=\frac{\pi^{2} E I}{L^{2}}
$$


Se um procedimento análogo ao empregado na determinação da carga crítica de Euler for usado para colunas com outros tipos de condição de vinculação em suas extremidades, é possível obter analiticamente suas cargas críticas. Na Figura 4 são apresentadas diferentes condições de vinculação para uma coluna e suas respectivas equações para a carga crítica.

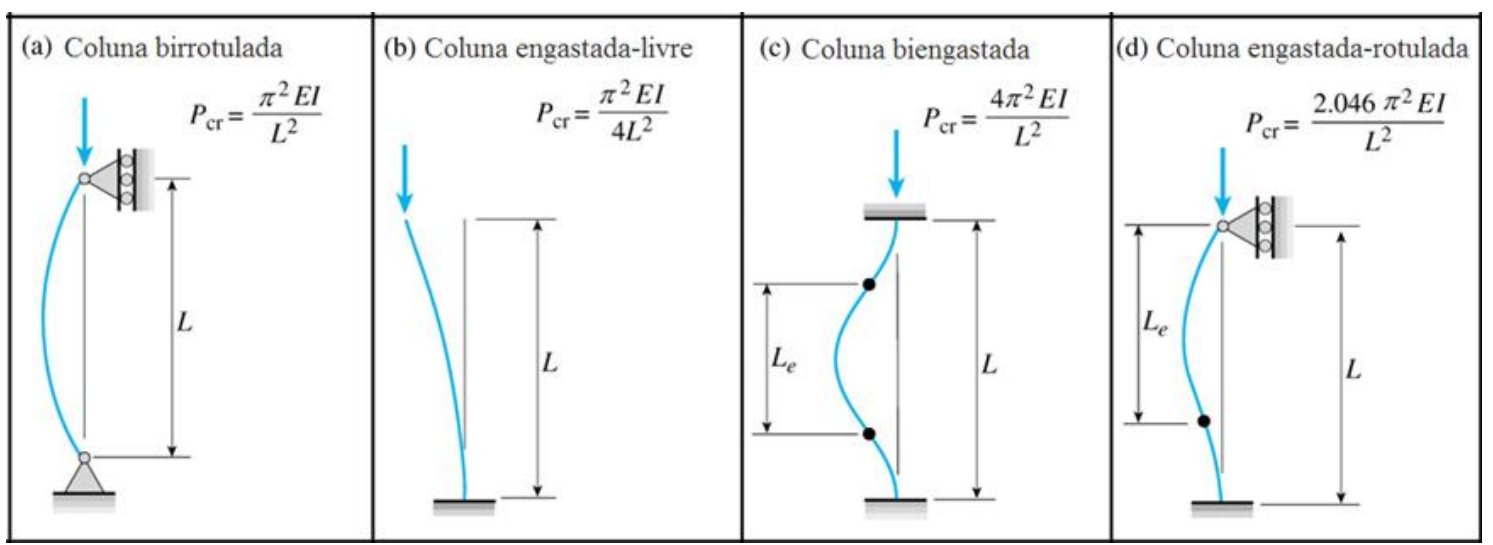

Figura 4: Carga crítica em colunas com diferentes tipos de condição de vinculação [4].

\section{MODELOS COMPUTACIONAIS}

Como antes mencionado, nesse trabalho foram desenvolvidos três modelos computacionais para tratar a flambagem de colunas. Fundamentalmente esses modelos diferem no tipo de elemento finito empregado. $O$ primeiro modelo, que emprega o elemento finito tipo viga (BEAM189), foi baseado em material publicado pela University of Alberta [5] que propôs um modelo computacional no software ANSYS para a coluna birrotulada. Então, a partir desse primeiro modelo computacional, outras condições de vinculação foram também consideradas no presente trabalho (ver Figura 4). Além disso, foram desenvolvidos dois novos modelos numéricos empregando elementos finitos tipo casca (SHELL93) e sólido (SOLID45).

Nos três modelos computacionais para a flambagem de colunas foi adotada a análise elástica de autovalores. As equações de equilíbrio por elementos finitos para este tipo de análise envolvem a solução de equações algébricas homogêneas cujo autovalor mais baixo corresponde à carga crítica de flambagem e o autovetor associado representa o modo primário de flambagem [5].

A formulação utilizada na análise numérica inclui tanto os termos lineares como os nãolineares. Assim, a matriz de rigidez total $[K]$, é obtida pela soma da matriz de rigidez convencional para pequenas deformações, $\left[K_{E}\right]$, com a matriz de rigidez geométrica, $\left[K_{G}\right]$. A matriz $\left[K_{G}\right]$ depende não só da geometria, mas também do esforço interno existente no início do carregamento, $\left\{P_{0}\right\}$. Então, a matriz de rigidez total da coluna para um nível de carga $\left\{P_{0}\right\}$ pode ser escrita como [6]:

$$
[K]=\left[K_{E}\right]+\left[K_{G}\right]
$$

Quando a carga atinge o nível de $\left\{P_{0}\right\}=\lambda\left\{P_{0}\right\}$, onde $\lambda$ é um escalar, a matriz de rigidez pode ser definida como:

$$
[K]=\left[K_{E}\right]+\lambda\left[K_{G}\right]
$$

sendo $\lambda$ um escalar. As equações de equilíbrio governantes para a coluna podem ser escritas como:

$$
\llbracket\left[K_{E}\right]+\lambda\left[K_{G}\right]\{U\}=\lambda\left\{P_{0}\right\}
$$

sendo $\{U\}$ o vetor de deslocamento total, que pode, portanto, ser determinado por: 


$$
\left.\left.\{U\}=\llbracket K_{E}\right]+\lambda\left[K_{G}\right]\right]^{-1} \lambda\left\{P_{0}\right\}
$$

$\mathrm{Na}$ flambagem, o componente estrutural apresenta um grande crescimento nos deslocamentos sem crescimento da carga. Por definição matemática é possível determinar a matriz inversa como a matriz adjunta dividida pelo determinante dos coeficientes, então os deslocamentos $\{U\}$ tendem a infinito quando:

$$
\operatorname{det}\left[\left[K_{E}\right]+\lambda\left[K_{G}\right]\right]=0
$$

A Equação (14) representa um problema de autovalores, que quando resolvido gera o menor autovalor, que corresponde à carga crítica $\left\{P_{c r}\right\}=\lambda_{1}\left\{P_{0}\right\}$ em que ocorre flambagem. Além disso, o vetor de deslocamento associado $\{U\}$ define a forma do modo de flambagem. $\mathrm{O}$ problema de autovalores é resolvido usando o método numérico de Lanczos [7].

\subsection{Modelo computacional com o elemento BEAM189}

O elemento BEAM189 do software ANSYS possui formulação baseada na teoria de vigas de Timoshenko, onde as deformações devido aos esforços de cisalhamento são consideradas. Ele é um elemento de viga, quadrático, tridimensional, com três nós (Figura 5). Cada nó possui seis graus de liberdade, sendo três translações em $x, y$ e $z$ e três rotações em torno desses eixos [7].

O domínio computacional é representado simplesmente por uma linha reta que deve possuir o mesmo comprimento $(L)$ da coluna que se deseja simular numericamente. A geometria e as dimensões da seção transversal da coluna são definidas no menu Sections do software ANSYS. Já as condições de contorno, de acordo com o tipo de vinculação desejado, e o carregamento devem ser aplicados diretamente nos nós superior e inferior do domínio computacional.

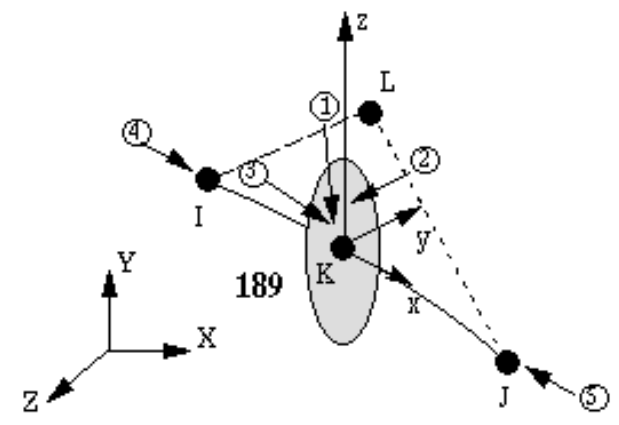

Figura 5: Elemento BEAM189 [7].

\subsection{Modelo computacional com o elemento SHELL93}

O elemento SHELL93 é indicado para modelar superfícies do tipo casca, possuindo oito nós e integração reduzida (Figura 6). Esse elemento tem seis graus de liberdade por nó: três translações nas direções dos eixos $x, y$ e $z$ e três rotações em torno dos eixos $x, y$ e $z$ [7].
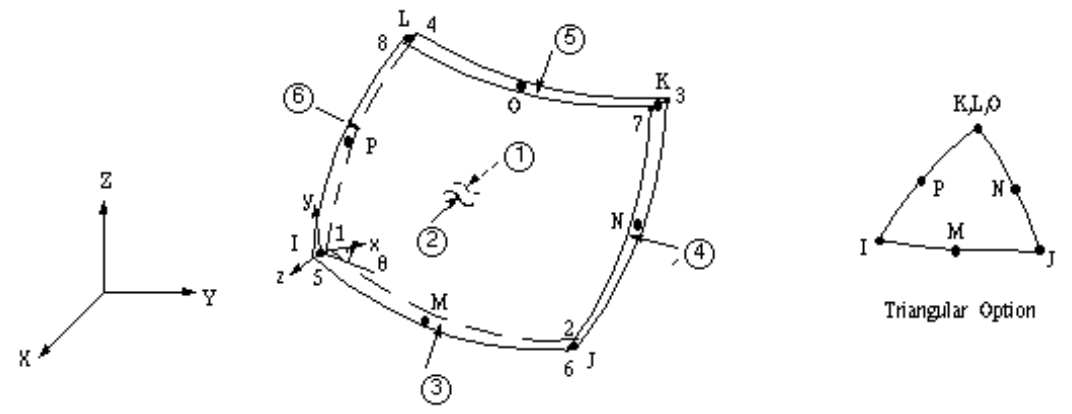

Figura 6: Elemento SHELL93 [7].

Nesse modelo, o domínio computacional também deve ter o mesmo comprimento da coluna a ser analisada. Entretanto a geometria e as dimensões da seção transversal da coluna deverão 
ser representadas por superfícies. Deve-se ainda informar a espessura das paredes da coluna, ou seja, a espessura que será considerada no elemento SHELL93, através do comando Real Constants. Além disso, como existem vários nós localizados nas extremidades do domínio computacional, e não somente um como no modelo anterior, as condições de contorno e as cargas devem ser aplicadas individualmente a cada um desses nós.

\subsection{Modelo computacional com o elemento SOLID45}

De acordo com [7], o elemento SOLID45 é um elemento finito tridimensional utilizado para a modelagem de estruturas sólidas, possuindo oito nós e tendo três graus de liberdade em cada um: translações nas direções $x, y$ e $z$ (Figura 7).

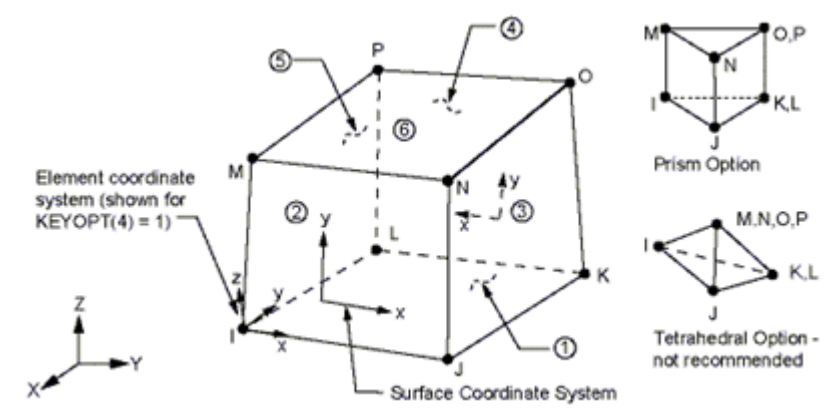

Figura 7: Elemento SOLID45 [7].

Para esse modelo numérico é necessário que o domínio computacional seja construído de maneira a representar completamente a coluna que se deseja analisar. Portanto, o comprimento da coluna, a geometria e as dimensões de sua seção transversal devem ser reproduzidas o mais fielmente possível. Assim como no modelo desenvolvido com o elemento SHELL93, as condições de contorno e as cargas devem ser aplicadas individualmente nos diferentes nós gerados nas extremidades do domínio computacional.

\section{RESULTADOS E DISCUSSÃO}

Um estudo de caso foi realizado considerando uma coluna de aço A-36 com módulo de elasticidade $E=210 \mathrm{GPa}$ e coeficiente de Poisson $v=0,3$, com $9 \mathrm{~m}$ de comprimento e possuindo a seção transversal indicada na Figura 8. Com cada modelo computacional desenvolvido duas diferentes condições de vinculação da coluna foram consideradas. Além disso, os resultados numéricos obtidos serão comparados com os resultados analíticos apresentados na seção 2. Essa comparação entre as soluções numéricas e analíticas para a flambagem da coluna servirá como verificação dos modelos computacionais desenvolvidos nesse trabalho.

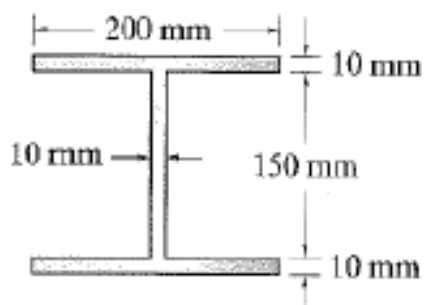

Figura 8: Seção transversal da coluna [1].

Considerando a Figura 4, para a coluna birrotulada a carga crítica de flambagem obtida analiticamente é $P_{c r}^{(a)}=340,32 \mathrm{kN}$; para a coluna engastada-livre é $P_{c r}^{(b)}=85,07 \mathrm{kN}$; para a coluna biengastada é $P_{c r}^{(c)}=1370,00 \mathrm{kN}$; e para a coluna engastada-rotulada a carga crítica analítica é $P_{c r}^{(d)}=696,30 \mathrm{kN}$. 


\subsection{Verificação do modelo computacional com o elemento BEAM189}

Considerando a coluna como birrotulada, as condições de contorno foram aplicadas nos nós extremos da coluna. No nó com coordenadas $(0,0,0)$ foram restringidas as translações em $x, y$ e $z$ e as rotações em torno de $x$ e $y$. E no nó com coordenadas $(9,0,0)$ as translações em $y$ e $z$ e as rotações em torno de $x$ e $y$ foram restringidas, e também foi aplicada a carga compressiva unitária (Figura 9a). O domínio computacional foi discretizado com três elementos e a configuração flambada da coluna pode ser observada na Figura 9b. A solução numérica determinou uma carga crítica $P_{c r}=342,16 \mathrm{kN}$ que em comparação com a solução analítica apresenta uma diferença de $0,54 \%$ foi encontrada.
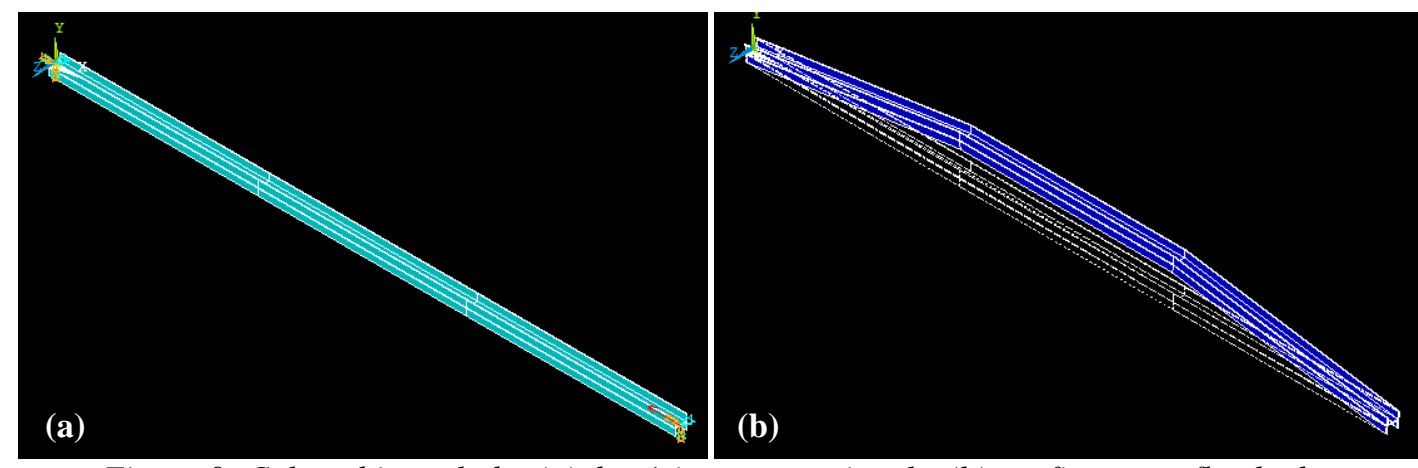

Figura 9: Coluna birrotulada: (a) domínio computacional e (b) configuração flambada.

Foi também analisada a coluna biengastada, que cujas condições de contorno no nó de coordenadas $(0,0,0)$ foram restringidas as translações na direções $x, y$ e $z$ e as rotações em torno dos eixos $x, y$ e $z$. Já no nó de coordenadas $(9,0,0)$ são restringidas a translações nos eixos $x$ e $y$ e as rotações em torno dos eixos $x, y$ e $z$. Nesse nó é também aplicada a carga de compressão de $1 \mathrm{~N}$ (Figura 10a). Aqui a coluna foi discretizada com cinco elementos e a forma assumida após a flambagem é apresentada na Figura 10b.
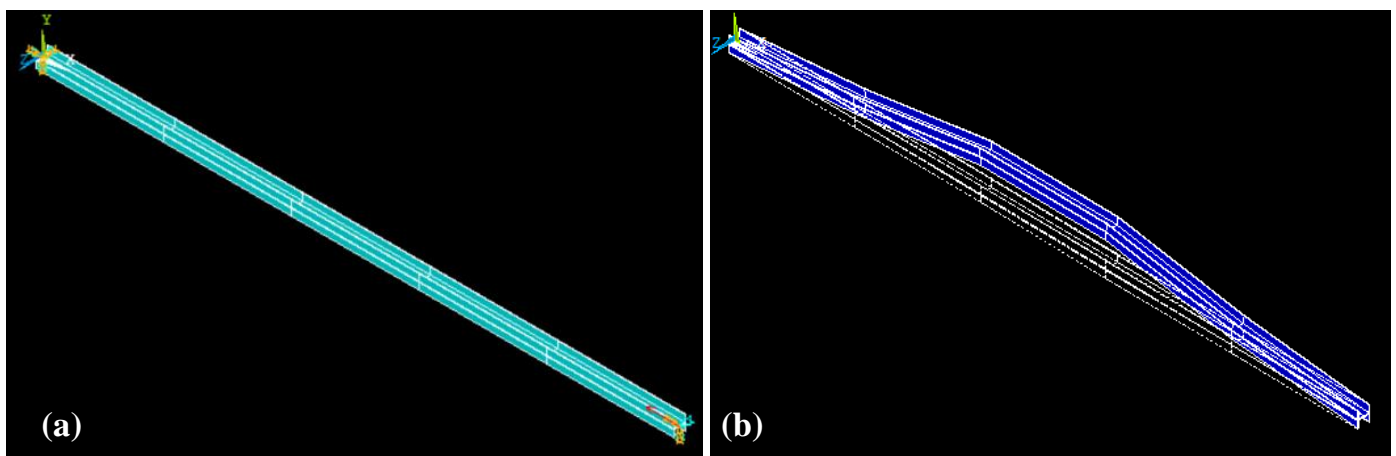

Figura 10: Coluna biengastada: (a) domínio computacional e (b) configuração flambda.

Foi obtido um resultado numérico para a carga crítica de $P_{c r}=1360,00 \mathrm{kN}$ possuindo uma diferença de $0,74 \%$ da solução analítica.

\subsection{Verificação do modelo computacional com o elemento SHELL93}

Aqui, novamente uma coluna birrotulada será analisada. Para a aplicação das condições de contorno, os nós centrais das extremidades do domínio computacional (detalhes em vermelho na Figura 11) podem possuir restrições diferentes das aplicadas aos demais nós (detalhes em amarelo na Figura 11). Nos nós centrais da extremidade inferior da coluna (em $x=0$ ) são restringidas as três translações $\left(u_{x}=u_{y}=u_{z}=0\right)$ e duas rotações $\left(r_{x}=r_{y}=0\right)$, enquanto nos demais nós é considerado $u_{y}=u_{z}=r_{x}=r_{y}=0$. Já na extremidade superior da coluna (em $x=$ $9 \mathrm{~m}$ ) em cada nó é imposto $u_{y}=u_{z}=r_{x}=r_{y}=0$ e aplicada uma carga compressiva de $-1 / 6 \mathrm{~N}$. Foi gerada uma malha regular com elementos finitos quadrilaterais com tamanho característico de 
$40 \mathrm{~mm}$. Assim, o modelo computacional empregando o elemento SHELL93 gerou um resultado de $P_{c r}=340,24 \mathrm{kN}$ com um diferença de apenas - $0,02 \%$ em relação à carga crítica analítica.

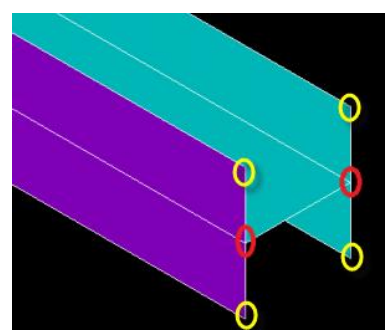

Figura 11: Nós na junção entre abas e alma da coluna.

Foi também simulada numericamente a coluna com vinculação engastada-livre. Nos seis nós da extremidade inferior $(x=0)$ foram restringidas todas as translações e rotações, enquanto nos nós da extremidade superior $(x=9 \mathrm{~m})$ somente a carga de $-1 / 6 \mathrm{~N}$ foi aplicada. A mesma discretização do caso anterior foi adotada. A carga crítica obtida numericamente foi de $P_{c r}=85,31 \mathrm{kN}$ que em comparação com a solução analítica apresenta uma diferença de $0,28 \%$.

\subsection{Verificação do modelo computacional com o elemento SOLID45}

Empregando o modelo computacional desenvolvido com o elemento SOLID45, inicialmente, foi considerada uma coluna engastada-livre. A aplicação das condições de contorno na extremidade inferior da viga $(x=0)$ foi realizada na área da seção transversal (Figura 11a) impedindo todas as translações e rotações. Na outra extremidade $(x=9 \mathrm{~m})$ somente a carga de compressão de $-1 / 2 \mathrm{~N}$ foi aplicada nos nós centrais destacados na Figura 11b. A discretização do domínio computacional foi realizada com elementos tetraédricos com tamanho característico de $20 \mathrm{~mm}$. O resultado numérico para a carga crítica foi de $P_{c r}=87,48 \mathrm{kN}$ que comparado com a solução analítica apresenta uma diferença $2,83 \%$.
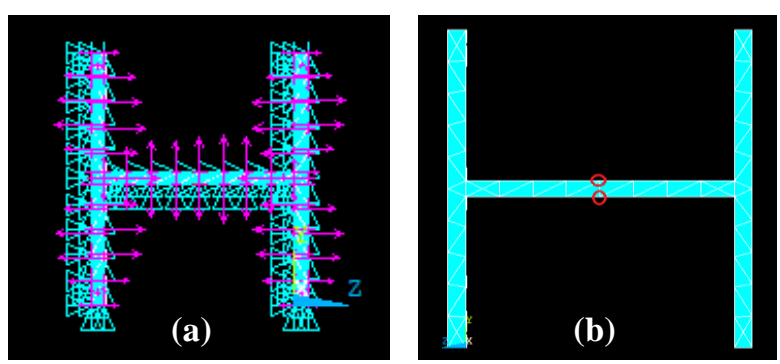

Figura 11: Domínio computacional: (a) aplicação das vinculações e (b) aplicação da carga

Por fim foi estudada a coluna com vinculação engastada-rotulada. Como no caso anterior foi considerado restringido os movimentos de translação e de rotação na área da seção transversal da extremidade inferior $(x=0)$ da coluna (ver Figura 11a). Já na outra extremidade $(x=9 \mathrm{~m})$, somente os movimentos de translação em $y$ e $z$ e as rotações em torno de $x$ e $z$ foram restringidos. Além disso o carregamento foi aplicado em 12 nós, conforme indicado na Figura 12 , com magnitude igual a $-1 / 12 \mathrm{~N}$ em cada um deles. A mesma discretização do caso anterior foi usada. O resultado numérico obtido foi de $P_{c r}=705,29 \mathrm{kN}$, possuindo uma diferença $1,39 \%$ em relação à solução analítica.

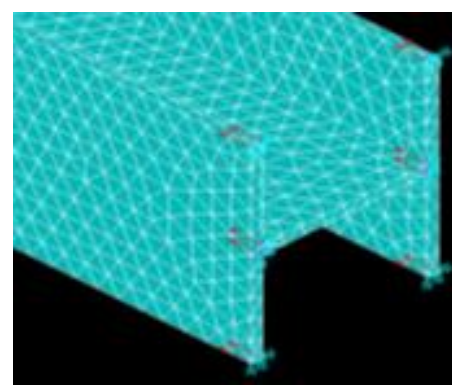

Figura 12- Distribuição do carregamento 


\section{CONCLUSÃO}

Este trabalho apresentou três diferentes modelagens computacionais para simular numericamente o problema da flambagem elástica em colunas. Esses modelos foram desenvolvidos no software ANSYS, que é baseado no Método dos Elementos Finitos (MEF). A diferença entre os modelos está justamente no tipo de elemento finito empregado na discretização do domínio computacional, originando diferenças na construção do domínio computacional, na imposição das condições de contorno e na aplicação do carregamento.

Os elementos finitos BEAM189, SHELL93 e SOLID45 foram usados, permitindo reproduzir diferentes condições de vinculação da coluna. Todos os modelos computacionais apresentaram resultados satisfatórios na determinação da carga crítica de flambagem da coluna bem como na definição da configuração flambada da coluna. Esses resultados numéricos foram comparados com resultados analíticos consagrados para esse tipo de problema de mecânica dos materiais. A diferença máxima encontrada foi de $2,83 \%$ o que verifica os modelos computacionais desenvolvidos.

Cabe destacar que os modelos computacionais desenvolvidos com os elementos SHELL93 e SOLID45 permitem a análise de situações práticas onde colunas que possuam seções transversais com geometrias complexas ou que possuam furos. Para esses casos, normalmente, não existem soluções analíticas e análises experimentais podem se tornar muito onerosas, sendo a simulação numérica uma alternativa confiável para a análise do comportamento mecânico dessas estruturas.

Em trabalhos futuros o modelo computacional que adota o elemento SHELL93 será usado para analisar a flambagem elástica de colunas com furos, uma vez que o mesmo permite análises com geometrias de maior complexidade, como comentado anteriormente, com um tempo de processamento menor se comparado ao modelo computacional que emprega o elemento SOLID45.

\section{AGRADECIMENTOS}

Os autores agradecem ao CNPq pelo apoio.

\section{REFERÊNCIAS BIBLIOGRÁFICAS}

1. Hibbeler RC. Resistência dos materiais. 7. ed. São Paulo: Pearson Prentice Hall, 2010.

2. Galambos TV, Surovek AE. Structural Stability of Steel: Concepts and Applications for Structural Engineers. New York. John Wiley \& Sons, 2008.

3. Chajes A. Principles of structural stability theory. Englewood Cliffs, New Jersey. Prentice Hall, 1974.

4. Gere JM. Mecânica dos materiais. Tradução Luiz Fernando de Castro Paiva. Revisão Técnica Marcio Lucio Bittencourt. São Paulo: Cengage Learning, 2009.

5. University of Alberta. ANSYS Intermediate Tutorials. Disponível em: http://www.mece.ualberta.ca/tutorials/ansys/IT/Buckling/Buckling.html.

6. Madenci E, Guven I. The Finite Element Method and Applications in Engineering Using ANSYS®. Ed. Springer, 2006.

7. ANSYS User's Manual (version 10.0), 2005. Swanson Analysis System Inc, Houston. 